\title{
Disturbed EEG sleep, paranoid cognition and somatic symptoms identify veterans with post-traumatic stress disorder
}

\author{
Harvey Moldofsky, Lorne Rothman, Robert Kleinman, Shawn G. Rhind and J. Donald Richardson
}

\section{Background}

Chronic post-traumatic stress disorder (PTSD) behavioural symptoms and medically unexplainable somatic symptoms are reported to occur following the stressful experience of military combatants in war zones.

\begin{abstract}
Aims
To determine the contribution of disordered EEG sleep physiology in those military combatants who have unexplainable physical symptoms and PTSD behavioural difficulties following war-zone exposure.
\end{abstract}

\section{Method}

This case-controlled study compared 59 veterans with chronic sleep disturbance with 39 veterans with DSM-IV and clinicianadministered PTSD Scale diagnosed PTSD who were unresponsive to pharmacological and psychological treatments. All had standardised EEG polysomnography, computerised sleep EEG cyclical alternating pattern (CAP) as a measure of sleep stability, self-ratings of combat exposure, paranoid cognition and hostility subscales of Symptom Checklist-90, Beck Depression Inventory and the Wahler Physical Symptom Inventory. Statistical group comparisons employed linear models, logistic regression and chi-square automatic interaction detection (CHAID)-like decision trees.

\section{Results}

Veterans with PTSD were more likely than those without PTSD to show disturbances in non-rapid eye movement (REM) and
REM sleep including delayed sleep onset, less efficient EEG sleep, less stage 4 (deep) non-REM sleep, reduced REM and delayed onset to REM. There were no group differences in the prevalence of obstructive sleep apnoeas/hypopnoeas and periodic leg movements, but sleep-disturbed, non-PTSD military had more EEG CAP sleep instability. Rank order determinants for the diagnosis of PTSD comprise paranoid thinking, onset to REM sleep, combat history and somatic symptoms. Decision-tree analysis showed that a specific military event (combat), delayed onset to REM sleep, paranoid thinking and medically unexplainable somatic pain and fatigue characterise chronic PTSD. More PTSD veterans reported domestic and social misbehaviour.

\section{Conclusions}

Military combat, disturbed REM/non-REM EEG sleep, paranoid ideation and medically unexplained chronic musculoskeletal pain and fatigue are key factors in determining PTSD disability following war-zone exposure.

\section{Declaration of interest}

None.

\section{Copyright and usage}

(C) The Royal College of Psychiatrists 2016. This is an open access article distributed under the terms of the Creative Commons Non-Commercial, No Derivatives (CC BY-NC-ND) license.
The disabling behaviour symptoms of military combatants, who were involved in the Viet Nam war, provided the impetus to characterise those psychological disturbances as post-traumatic stress disorder (PTSD). This psychiatric diagnosis first appeared in the third edition of the APA's Diagnostic and Statistical Manual (DSM-III), with its seminal feature being a specific precipitating stressful event. Subsequently, two major revisions of the DSM better characterised PTSD's behavioural diagnostic features. There are three clusters of behaviour symptoms in DSM-IV ${ }^{1}$ and four cluster groups in DSM-5. ${ }^{2}$ These four clusters comprise reexperiencing, avoidance, negative mood and cognitions (such as depressive, hostile, aggressive and paranoid thinking), and behavioural arousal including sleep disturbances. In addition to its behavioural features, military medical personnel have described a variety of chronic disabling physical symptoms following exposure to combat. ${ }^{3}$ These troublesome physical complaints lack any objective physical pathology. Where the focus is on unexplained widespread variable musculoskeletal pain and chronic fatigue, the current preferred diagnosis is fibromyalgia (formerly known as rheumatism or fibrositis). This diagnosis overlaps with equally medically unexplained syndromes such as Gulf War syndrome, chronic fatigue syndrome, combat fatigue (with previous labels including Da Costa's syndrome, disordered action of the heart, neurasthenia or neuro-circulatory asthenia, and effort syndrome). Patients with fibromyalgia have unrefreshing sleep with disturbances in EEG sleep physiology. Moreover, experimentally induced sleep physiological disturbances in normal individuals and animals result in increased pain sensitivity and pain symptoms. Clinical and epidemiological studies show that chronic sleep disturbances are integral to a vicious self-perpetuating cycle of chronic pain, fatigue, negative mood and cognition in patients with fibromyalgia. These symptoms may be precipitated by a distressing traumatic industrial or motor vehicle accident. ${ }^{4}$ Therefore, the purpose of this study was to determine whether the disordered physiology of the sleeping/ waking brain may provide the link between the behavioural and somatic symptoms of those military personnel experiencing postcombat chronic PTSD. The analyses comprised a two-part strategy: first, to establish differences between treatment-resistant PTSD and non-PTSD military personnel in multiple domains comprising EEG sleep physiology, behavioural and the medically unexplained pain and fatigue somatic symptoms; and second, to generate a decisiontree probability model for PTSD based on these differences. That is, the key behavioural elements that distinguish chronic PTSD from non-PTSD military comprise a specific stressful traumatic event (i.e. combat), altered sleep EEG physiology, persistent unexplained somatic symptoms and negative cognitive behaviour.

We employed a case-controlled study of PTSD in which both case and control groups included veterans with and without 
experience in combat zones, enabling us to separate PTSD symptoms from those that might be associated with combat experience alone and to assess combat experience along with other patient measures when building models. We measured behaviour symptoms, including negative cognition (depressive, hostile, aggressive and paranoid thinking), and chronic physical health concerns, including disturbances in EEG sleep physiology, sleep quality and medically unexplained somatic symptoms (e.g. musculoskeletal pain and fatigue).

\section{Method}

\section{Participants}

The participants comprise consecutive patients referred between 2008 and 2014 from an Operational Stress Injury (OSI) clinic of the Department of Veteran Affairs of Canada. The OSI clinic specialises in the assessment and treatment of PTSD and other operational stress injuries in the Canadian military and veteran forces (CF). The diagnosis of PTSD was determined by a psychiatrist employing a comprehensive psychiatric examination and the Clinician-Administered PTSD Scale (CAPS) for DSM-IV, ${ }^{5}$ where the CAPS mean rating was 77.38 and s.d. 21.36. To control for commonly disrupted and insufficient sleep problems in the military, we compared sleep and symptoms in CF veterans with persistent symptoms of PTSD with CF personnel without a history PTSD who complained of chronic insomnia. This latter group of participants were referred from Canadian Department of National Defence (DND) medical ambulatory clinics for diagnostic assessment of a possible chronic primary sleep disorder such as sleep apnoea or restless legs/sleep-related periodic limb movement disorder.

The participants consented to be assessed in the Centre for Sleep and Chronobiology in Toronto for specialised comprehensive sleep/wake clinical and laboratory investigations of their chronic treatment-resistant symptoms. The research was reviewed and approved by the DND, Institute of Military and Veterans Research Institute in association with Defence Research and Development Canada.

Table 1 shows the description of the participants: gender, mean age, mean BMI and the independent variables: combat experience (combat $1=$ yes and $0=$ no or unknown) and the presence of treatment-resistant PTSD ( $1=$ yes and $0=$ no) were independent variables. All patients with PTSD (33 with combat and 6 without) were referred from the OSI. No patients with PTSD were referred from the DND (28 with combat and 31 without). There were no significant differences in the two groups' ages and BMI values. There were no females with PTSD in the study. SAS Software Multivariate Analysis of Variances (MANOVAs) tests showed no significant gender differences when comparing non-PTSD females $(n=9)$ with non-PTSD males $(n=50)$. Both genders were included in the study. Combat experience included those patients who had served in combat zones (e.g. Gulf War and Afghanistan) and those who developed treatment-resistant PTSD symptoms following deployment to the so-called 'U.N. peace-keeping missions' in dangerous combative environments, that is, the Balkans (Bosnia and Croatia) and Africa/Middle East (Suez Canal zone, Rwanda, Somalia and Cyprus). Six cases of PTSD occurred in sleepdisturbed, non-combat CF patients. Distressing non-combat traumatic events were quite varied. They included sexual assault, witness to a major airplane crash, engagement in dangerous service with the military police, witness to a suicide of a soldier and participation in a military training exercise.

All patients with treatment-resistant PTSD $(n=39)$ were functionally impaired despite psychological and lengthy pharmacological treatments (median 32.5 months (range 6-249 months)).

All patients with PTSD had received cognitive-behavioural treatments, which included trauma-focussed behaviour therapy such as prolonged exposure and various types of psychological support, for example, individual, marital, family counselling and group therapies.

At the time of the sleep study, patients with PTSD were taking at least two drug class combinations of antipsychotics (e.g. risperidone, quetiapine, aripiprazole; $n=12$ ), various antidepressants (e.g. mirtazapine, bupropion, venlafaxine, citalopram; $n=34$ ), anticonvulsant mood modulators (sodium valproate or topiramate; $n=13$ ), and benzodiazepine sedative hypnotics $(n=8)$. In comparison with the patients with PTSD, the 59 with unremitting sleep problems and no PTSD used fewer antidepressants $(n=16)$, anticonvulsants $(n=4)$, antipsychotics $(n=5)$ and sedative hypnotics $(n=1)\left(\right.$ all $\chi^{2}$ test, $\left.P<0.05\right)$. Table 1 provides a detailed description of the groups of participants, with and without PTSD, their use of classes of medications and whether or not they had been exposed to combat. The medical records that were made available did not specify the psychotropic medications before their treatments at the time of the polysomnographic sleep study. In particular, of the unknown number of individuals who had previously received the alpha1-adrenergic receptor blocker (prazosin) for disturbing dreams, only two participants continued to find that this drug satisfactorily reduced their recurrent nightmares. Moreover, the psychotropic treatments were not withdrawn to avoid potential adverse effects and permit full cooperation of the patients to participate in the various physiological and behavioural proceedings.

In addition, to the psychotropic drugs shown in Table 1, other medications that were consumed include the following.

- Patients with PTSD received pain medication (opiates: 3; NSAIDs: 6). Sixteen patients received medication for

\begin{tabular}{|c|c|c|c|c|}
\hline & \multicolumn{2}{|c|}{ PTSD - yes } & \multicolumn{2}{|c|}{ PTSD - NO } \\
\hline & Combat - yes & Combat - no & Combat - yes & Combat - no \\
\hline Total number of participants & 33 & 6 & 28 & 31 \\
\hline Number of females & 0 & 0 & 2 & 7 \\
\hline Number of males & 33 & 6 & 26 & 24 \\
\hline Mean age (years) & 44.4 & 46.8 & 40.5 & 41.6 \\
\hline Mean BMl & 30.1 & 32.5 & 30.1 & 32.5 \\
\hline \multicolumn{5}{|l|}{ Drugs } \\
\hline Antipsychotics & 9 & 3 & 2 & 3 \\
\hline Antidepressants & 27 & 7 & 5 & 11 \\
\hline Anticonvulsants & 10 & 3 & 2 & 2 \\
\hline Benzodiazepines & 8 & 0 & 0 & 1 \\
\hline Prazosin & 2 & 0 & 0 & 0 \\
\hline
\end{tabular}


cardiovascular problems. Three patients were treated for type 2 diabetes.

- Those without PTSD were prescribed more NSAIDs $(n=11)$ and no opiates, but similar cardiovascular drugs $(n=13)$ and antidiabetic drugs $(n=4)$.

Those individuals who had been previously identified by the military physicians as having problems with alcohol and/or substance abuse/dependency had been treated for their condition(s) before the time of referral for their sleep study. For this study, all participants completed a self-rated standardised questionnaire on the types, amount and frequency of use of alcohol and recreational drugs over the previous year and during 2 weeks before their sleep study. During the 2 weeks before the sleep study, neither group abused alcohol and there were no differences in their alcohol habits. During the previous year, eight patients with PTSD used cannabis, whereas none of the patients without PTSD used cannabis $\left(\chi^{2}\right.$ test, $\left.P<0.01\right)$. Within the 2 weeks before the sleep study, only two patients with PTSD had occasionally taken cannabis drugs, whereas none in the sleep-disordered group used cannabis drugs ( $\chi^{2}$ test, $P=\mathrm{NS}$ ).

The individuals with and without PTSD completed psychological and physical symptom self-reports before a single overnight EEG sleep study. Because sleep disturbance has the potential to promote negative cognitions such as irritability, aggressive behaviour, paranoid and depressive thinking that may occur with PTSD, ${ }^{6-8}$ we employed the Symptom Checklist-90 (SCL-90) subscales for hostility and paranoid thinking ${ }^{8,9}$ and the Beck Depression Inventory (BDI). ${ }^{10}$ Physical symptoms were self-rated from 1 to 5 with the Wahler Physical Symptom Inventory (WPSI). ${ }^{11,12}$ The number of symptoms self-rated as 4 or 5 on the WPSI scale (corresponding to 'about twice a week' or 'nearly every day') was tallied to determine the prevalence of troublesome symptoms. Frequency of regional pain symptoms, ${ }^{13}$ severity of fatigue (range from 1 'full of energy' to 7 'totally physically exhausted' $)^{14}$ and Stanford Sleepiness Scale ${ }^{15,16}$ were self-rated before and after the overnight sleep.

The frequency of sleep-related behavioural disturbances during the previous 2 months was rated on the Sleep-Wake questionnaire with a graded scale of $0-3(0=$ never, $1=$ sometimes, $2=$ often and $3=$ always). The behavioural disturbances during sleep included frequency of awakening during the night because of nightmares or unpleasant dreams, told that you had screamed or shouted and disturbing (bad) dreams during sleep. Abnormal cognition included self-ratings of frequency of harmful behavioural to self and others noted in BDI Q9 (10): thoughts of killing self-rated on a scale of 0-3. Abnormal temper outbursts that could not be controlled included urges to beat, injure or harm someone; urges to break or smash things; shouting or throwing things (questions from the SCL-90, which were rated on a scale of $0-4: 0=$ not at all, $1=\mathrm{a}$ little bit, $2=$ moderately, $3=$ quite a bit, $4=$ extremely). ${ }^{11}$

Physiological assessment of sleep comprised standardised EEG polysomnography (PSG). The PSG included electroencephalogram (EEG C3 and C4), electro-oculogram, submental and bilateral anterior tibialis electromyogram, single anterior lead electrocardiogram, measures of respiration comprising measures of airflow with oral-nasal thermistors, respiratory impedance plethysomography and pulse oximetry. An experienced registered PSG technologist completed blind ratings of sleep physiological indices. ${ }^{17,18}$ The sleep EEG stages were scored according to the original Rechtschaffen and Kales criteria, ${ }^{17}$ which were needed for a computerised physiological sleep measure of EEG sleep stability known as the Cyclical Alternating Pattern (CAP). ${ }^{19-21}$ Sleep EEG CAP is characterised by sequences of transient EEG changes in non-REM sleep that occur distinctively from the background
EEG activities. They comprise three subtypes: $\mathrm{CAP}_{\mathrm{A} 1}$ is related to favourable sleep stability. $\mathrm{CAP}_{\mathrm{A} 2}$ and $\mathrm{CAP}_{\mathrm{A} 3}$ are progressive indices of poor sleep quality or sleep instability. ${ }^{19}$ These abnormal physiological features have been observed in patients who have chronic widespread musculoskeletal pain and fatigue, ${ }^{22,23}$ as well as sleep-related breathing disorders. ${ }^{24}$ Analysis of nocturnal sleep EEG CAP was determined by an Embla computerised automatic detection system. ${ }^{21,22}$

\section{Data analyses}

MANOVAs were fit into four groups of dependent variables. These included PSG and sleep EEG CAP indices; self-ratings of behaviour, including negative cognition; pre- and post-PSG sleepiness and fatigue; and physical symptom severity. Combat experiences (Combat $1=$ yes, $0=$ no or unknown) were the independent variables that were compared to whether or not they had treatment-resistant PTSD (PTSD $1=$ yes, $0=$ no) (see Table 1 ). Because there were only six patients with $\mathrm{PTSD}=1$ and Combat $=0$, we did not test for PTSD $\times$ Combat experience interactions. EEG sleep measures included sleep architecture and computerised measure of EEG sleep stability, that is, sleep EEG CAP rate. MANOVA permitted the testing of the significance of suites of measures, while accounting for relationships among the dependent variables as well as the relationships between independent and dependent variables. Initial data exploration showed skewed distributions, and outliers, particularly for the sleep physiology measures. Normality assumptions were likely violated. For significance testing, MANOVA and ANOVA models were fitted to ranked measures; tests on ranked data provided nonparametric tests. ${ }^{25-28}$ All inferences were based on these ranked nonparametric tests. Because multiple testing inflates Type 1 error, or the probability of rejecting the null hypothesis when true, $P$-values from ANOVAs were adjusted for multiple testing within each of the four independent variable groups by the adaptive step-down Holm method. ${ }^{29}$ This method controlled for the error rate within a family of tests (family-wise error). The test does not assume independence between the various dependent variables in these ANOVAs.

We used three methods to identify distinguishing characteristics of PTSD and non-PTSD patients: canonical discriminant analysis (CDA), logistic regression and decision trees. PTSD was the dependent variable. Patient measures and combat experience were the independent variables.

We fitted a chi-square automatic interaction detection (CHAID)like decision-tree algorithm by SAS Enterprise Miner. ${ }^{28}$ The CHAID approach finds splitting rules that create pure segments of data by a chi-squared test of association with a significance cut-off (alpha of $0.05)$, where all $P$-values are adjusted for multiple testing by a Bonferroni method. Missing values were addressed through multiple imputations by the predictive mean matching method, which is more appropriate for non-normal measures. ${ }^{29,30}$ BDI was excluded from decision-tree and regression modelling because this self-rated test includes both somatic symptoms such as sleep, pain and fatigue, and abnormal cognitive behavioural data.

\section{Results}

Patients with PTSD differed from patients without PTSD in prevalence of suites of physical and behavioural health problems, while controlling for the effects of combat exposure. MANOVA tests showed significant differences between patients with PTSD and without PTSD on sleep measures $(P<0.01)$, psychological symptoms self-reports $(P<0.0001)$ and physical symptoms selfreports $(P<0.01)$. The MANOVA test showed no significant difference between PTSD and non-PTSD on subtypes of sleep EEG CAP measures $(P>0.05)$. 
While controlling for the effects of combat exposure, individual ANOVAs showed differences between PTSD and non-PTSD groups in their distributions of onset to EEG sleep, sleep efficiency, EEG stage 4\%, REM sleep onset latency, REM\% and EEG CAP indices measures including CAP frequency, $\mathrm{CAP}_{\mathrm{A} 2}$, $\mathrm{CAP}_{\mathrm{A} 3} / \mathrm{CAP}_{\mathrm{A} 1+\mathrm{A} 2+\mathrm{A} 3}{ }^{22}$ (see Table 2). Those with PTSD took longer to fall asleep, had less efficient sleep (i.e. less time asleep relative to time in bed), less deep (stage 4) sleep, lower percent of time in REM sleep and a prolonged delay to onset of REM sleep. The higher CAP frequency index in the non-PTSD group is consistent with their complaint of chronic poor quality of sleep in the absence of a specific combat-related traumatic experience. These sleep EEG differences may be influenced by more frequent use by the PTSD group of psychotropic drugs.

There were no group differences in prevalence of primary sleep disorders such as obstructive sleep apnoea or sleep-related periodic leg movements (PLM). The prevalence of patients with PTSD with an index of obstructive sleep apnoeas/hypopnoeas per hour of sleep $>5$ per hour $(n=35)$ did not differ from patients without PTSD $\left(n=25, \chi^{2}=\mathrm{NS}\right)$. Furthermore, there were no statistical differences in their frequencies of sleep EEG arousals from sleep apnoea/ hypopnoeas or with sleep-related PLM (see Table 2).

Behavioural disturbances during sleep were more common among the PTSD veterans. Of the 39 patients with PTSD, 32 were more likely to be awakened by nightmares versus 11 of 59 patients without PTSD ( $\chi^{2}$ test, $\left.P<0.05\right)$. Twenty-one patients with PTSD versus six patients without PTSD reported screaming or shouting during sleep ( $\chi^{2}$ test, $P<0.05$ ). Seven of those in the combat-related PTSD group also reported inadvertently striking, but not seriously injuring, their partner during sleep, whereas none of the nonPTSD group described any abnormal sleep-related behaviour.

Patients with PTSD had greater mean ratings on the WPSI with more frequent physical problems (commonly musculoskeletal pain and post-sleep fatigue) (Table 3 ).

Patients with PTSD described significantly more BDI depressive symptoms (see Table 4). Further, seven patients with PTSD reported suicidal intentions, whereas none of the patients without PTSD reported suicidal intentions.

More patients with PTSD reported hostility and paranoid notions on the SCL-90 (Table 4). Nineteen participants reported domestic and/or violent social behaviour with three being charged and arrested for assaultive behaviour. In contrast, none of the non-combat non-PTSD patients reported any form of social misbehaviour $\left(\chi^{2}\right.$ test, $\left.P<0.001\right)$.

$\mathrm{CDA}$ and, in particular, logistic regression and decision trees identified the most salient combinations of measures that characterise PTSD. All five runs of CDA, which used multiply imputed data sets, showed significant discriminant functions $(P<0.001)$. Excluding combat exposure, the top six variables that are most correlated $(|r|>0.4)$ with these functions and were the most discriminating characteristics of PTSD, comprise the following features: BDI total score, paranoid score, REM onset latency, SCL-90 hostility scale score, WPSI symptom score and reduced REM\% sleep. Regression models that minimise Schwarz criteria reveal that PTSD is described by REM onset latency, paranoid score and combat exposure. Individuals with combat experience had 4.7 times the odds of having PTSD compared to those without combat exposure. Because selective serotonin reuptake inhibitor (SSRI) and serotonin-noradrenaline reuptake inhibitor (SNRI) antidepressants may cause a delay in REM onset latency ${ }^{31}$ and both antipsychotic as well as anticonvulsant neurotropic drugs may influence EEG sleep physiology, we also re-ran the selection routine while forcing these drugs as variables into the model. The selection routine chose the same variables. PTSD in these individuals is still described by REM onset latency, paranoid score and combat exposure, even while controlling for the use of antidepressant, antipsychotic and anticonvulsants.

The CHAID-like decision-tree analysis reveals the key traits that distinguish PTSD from non-PTSD and their relative importance. Paranoid score was the most important variable in the tree model, followed by REM onset latency, exposure to combat and WPSI severity score based on each measure's contribution to impurity reduction across the tree (relative importance: $1,0.79$, 0.77 and 0.49 , respectively). For individuals who were exposed to combat, longer delays to onset of REM sleep (REM onset latency $\geq 175.5 \mathrm{~min}$ ) lead to higher propensity of exhibiting PTSD $(0.79$ or $79 \%)$. Lower REM onset latency and high paranoid scores ( $\geq 7.5$ ) lead to high PTSD propensity $(0.857$ or $85.7 \%)$, whereas low paranoid scores $(<2.5)$ lead to low PTSD propensity. With no combat experience, PTSD is still highly probable $(0.83$ or $83 \%)$ with higher paranoid scores $(\geq 2.5)$ and higher somatic symptom ratings (WPSI $\geq 11$ ). On the other hand, those non-combat individuals with lower paranoid and somatic symptom ratings were likely to have a low propensity for PTSD (see Fig. 1).

\begin{tabular}{|c|c|c|c|c|c|c|c|}
\hline \multirow[b]{3}{*}{ Dependent } & \multicolumn{6}{|c|}{ PTSD } & \multirow[b]{3}{*}{$P$} \\
\hline & \multicolumn{3}{|c|}{ No } & \multicolumn{3}{|c|}{ Yes } & \\
\hline & LSM & SE & $n$ & LSM & SE & $n$ & \\
\hline Sleep onset latency (min) & 11.9 & 1.81 & 58 & 17.38 & 2.47 & 38 & $<0.05$ \\
\hline Sleep efficiency (\%) & 85.5 & 2.05 & 58 & 81.19 & 2.81 & 38 & $<0.05$ \\
\hline Stage 1 (\%) & 12.36 & 1.7 & 58 & 14.56 & 2.32 & 38 & NS \\
\hline Stage $2(\%)$ & 61.76 & 1.36 & 57 & 66.31 & 1.84 & 38 & NS \\
\hline Stage $3(\%)$ & 6.33 & 0.78 & 57 & 5.74 & 1.06 & 38 & NS \\
\hline Stage 4 (\%) & 2.01 & 0.44 & 56 & 0.91 & 0.61 & 36 & $<0.05$ \\
\hline REM (\%) & 19.14 & 0.93 & 57 & 11.66 & 1.26 & 38 & $<0.0001$ \\
\hline REM onset latency (min) & 124.91 & 10.61 & 56 & 205.9 & 14.69 & 35 & $<0.001$ \\
\hline Apnoea-hypopnoea arousals & 74.73 & 15.92 & 57 & 109.6 & 21.79 & 37 & NS \\
\hline PLM arousals & 17.57 & 5.16 & 57 & 32.98 & 7.27 & 36 & NS \\
\hline Spontaneous arousals & 51.48 & 4.62 & 58 & 45.25 & 6.38 & 37 & NS \\
\hline $\mathrm{CAP}_{\mathrm{A} 2}$ Index cycle & 7.07 & 0.74 & 56 & 5.49 & 1.01 & 37 & NS \\
\hline $\mathrm{CAP}_{\mathrm{A} 3}$ Index Cycle & 9.16 & 0.99 & 56 & 6.01 & 1.34 & 37 & NS \\
\hline $\mathrm{CAP}_{\mathrm{A} 1} / \mathrm{CAP}_{\mathrm{A} 1+\mathrm{A} 2+\mathrm{A} 3}$ & 0.25 & 0.02 & 56 & 0.24 & 0.03 & 37 & NS \\
\hline Total CAP Index Cycle & 74.77 & 4.23 & 56 & 57.83 & 5.75 & 37 & $<0.05$ \\
\hline
\end{tabular}




\begin{tabular}{|c|c|c|c|c|c|c|c|}
\hline \multirow[b]{3}{*}{ Dependent } & \multicolumn{6}{|c|}{ PTSD } & \multirow[b]{3}{*}{$P$} \\
\hline & \multicolumn{3}{|c|}{ No } & \multicolumn{3}{|c|}{ Yes } & \\
\hline & LSM & SE & $n$ & LSM & SE & $n$ & \\
\hline Pre-sleep sleepiness & 2.94 & 0.14 & 53 & 2.99 & 0.20 & 33 & NS \\
\hline Post-sleep sleepiness & 3.03 & 0.14 & 51 & 3.27 & 0.19 & 34 & NS \\
\hline Pre-sleep fatigue & 3.67 & 0.20 & 53 & 4.38 & 0.28 & 33 & NS \\
\hline Post-sleep fatigue & 2.93 & 0.18 & 51 & 3.88 & 0.24 & 34 & $<0.01$ \\
\hline Pre-sleep pain & 3.02 & 0.51 & 52 & 5.25 & 0.71 & 34 & NS \\
\hline Post-sleep pain & 3.19 & 0.57 & 51 & 5.16 & 0.78 & 34 & NS \\
\hline Somatic symptoms (WPSI) & 5.44 & 0.63 & 57 & 10.15 & 0.88 & 37 & $<0.001$ \\
\hline
\end{tabular}

All $P$-values from ranked (non-parametric) tests are adjusted by the adaptive Holm method to control family-wise error rate. Least-square means (LSM) for PTSD, no (0) and yes (1), are adjusted to remove combat effect. Bold denotes significance.

\section{Discussion}

This study provides provisional evidence for the key clinical and physiological sleep features that characterise veterans who remain functionally disabled with PTSD despite various drug and behavioural treatments. The results are consistent with DSM-V behavioural symptomology. The association of malfunctions of the sleeping/waking brain and medically unexplained physical symptoms with DSM-IV behavioural symptoms, identified in the MANOVA and CDA analyses, broadens our understanding of chronic PTSD. These psychological and somatic symptoms are consistent with our hypothesis that EEG sleep disturbances may provide the link between negative cognition and mood, and somatic symptoms. While ANOVA and MANOVA statistics show these symptoms to be independent of combat stressor effects, the warzone experience itself is a key characteristic of PTSD among military personnel, as shown by both the logistic regression and decision-tree models (see Fig. 1). Our decision-tree model in Fig. 1 shows that combat military service, disturbed REM sleep, paranoid thinking and medically unexplainable somatic symptoms (commonly musculoskeletal pain) characterise CF veterans with chronic PTSD. However, the regression model, while identifying combat and altered EEG sleep, did not include somatic symptoms.

Our EEG sleep results complement previous studies that show the importance of disturbances in REM and non-REM sleep. Those with chronic PTSD take longer to fall asleep, have reduced sleep efficiency, reduced stage 4 (deep) sleep, reduced and delayed onset to REM sleep. ${ }^{32-35}$ A meta-analysis of previous polysomnographic studies of military and civilian patients with PTSD versus those without PTSD showed similar findings with more stage 1 (light) sleep, less slow-wave (deep) sleep and alterations in REM. ${ }^{34}$ Furthermore, our findings are consistent with previous PTSD sleep studies, which show prolongation in the onset to REM sleep. ${ }^{35,36}$ The higher EEG CAP frequency in the patients without PTSD, a sensitive marker for sleep instability, ${ }^{19}$ may be related to their primary complaint of disturbed and unrefreshing sleep. Such differences in EEG CAP frequency are unlikely to be affected by psychotropic drugs, which reduce EEG CAP rate. ${ }^{37}$ Unlike previous studies that associate sleep-related breathing disorders to sleep EEG CAP ${ }^{24}$ and to PTSD in the military, ${ }^{35}$ this study found no statistical differences in apnoea/hypoponea index, sleep-related respiratory arousals or frequency of periodic limb movement arousals between those with combat-related PTSD and those with sleep-disturbed, non-PTSD military individuals.

In this study of CF veterans with chronic post-combat PTSD, a prolonged delay in the onset to REM sleep is accompanied by negative mood and negative cognitive symptoms. Whereas most tricyclic, SSRI and SNRI antidepressant drugs may reduce and delay the onset to REM sleep, ${ }^{31}$ the delay in onset to REM sleep persists when the use of antidepressants, as well as anticonvulsants and psychotropic drugs, is statistically controlled. A previous sleep EEG study, however, that compared the sleep of drug-free combatrelated PTSD patients with patients with major depression and normal individuals showed that the PTSD group had similar reduction in REM sleep and no delay in onset to REM sleep. ${ }^{36}$ Overall, our results are supportive of Germain's hypothesis and previous EEG sleep research that disturbed REM and non-REM sleep contribute to maladaptive stress and trauma responses in PTSD. ${ }^{38}$

Ideally, further studies of sleep physiology should involve comparisons between a group of non-sleep-altering medicated sleep-disturbed individuals with PTSD and age-matched normal healthy sleepers who are acclimatised to the novelty of the laboratory environment. To affirm the importance of a disorganisation of the REM/non-REM sleep as a possible key factor to the emergence of PTSD, it would be preferable to examine the EEG sleep before any drug administration, which may influence sleep physiology. The design of the research did not permit a comparative study with those who had benefitted from specific pharmacological and/or behavioural treatment. A future study employing similar physiological and behavioural methodology should comprise a large group of randomised, early-identified combat-exposed versus noncombat-exposed individuals who are free of potentially sleep EEG confounding psychotropic drugs. Such research may pave the way to determining what pharmacological and behavioural methods would be useful in those early-detected post-combat military with PTSD.

Our decision to include measures of negative cognition and mood in patients with PTSD, diagnosed by DSM-IV criteria,

\begin{tabular}{|c|c|c|c|c|c|c|c|}
\hline \multirow[b]{3}{*}{ Dependent } & \multicolumn{6}{|c|}{ PTSD } & \multirow[b]{3}{*}{ P } \\
\hline & \multicolumn{3}{|c|}{ No } & \multicolumn{3}{|c|}{ Yes } & \\
\hline & LSM & SE & $n$ & LSM & SE & $n$ & \\
\hline Hostility score & 3.02 & 0.63 & 53 & 6.56 & 0.84 & 36 & $<0.001$ \\
\hline Paranoid score & 2.05 & 0.48 & 56 & 5.68 & 0.66 & 35 & $<0.0001$ \\
\hline BDI Ss & 9.88 & 1.05 & 56 & 22.74 & 1.47 & 36 & $<0.0001$ \\
\hline
\end{tabular}




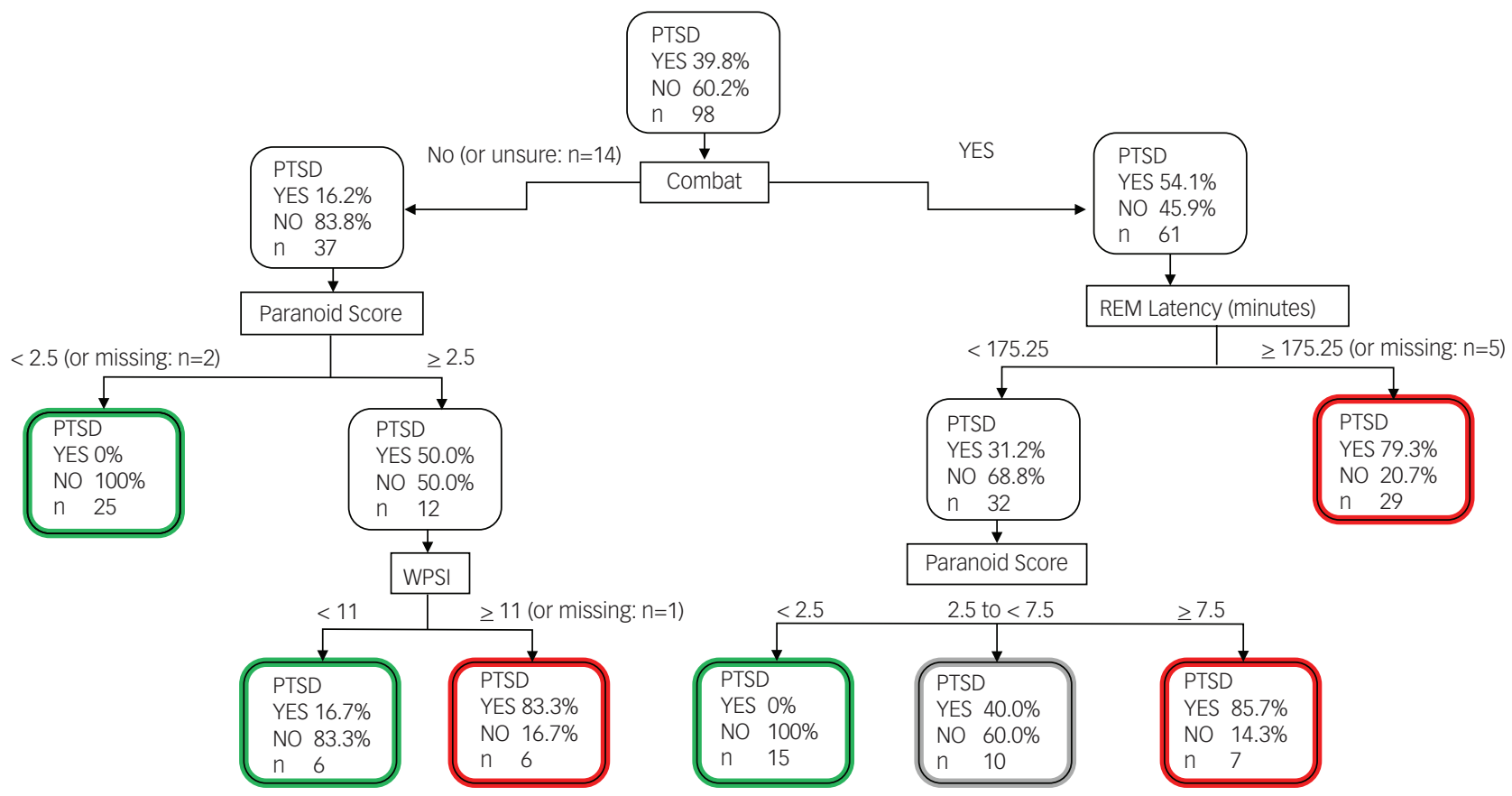

Fig. 1 Chi-square automatic interaction detection (CHAID)-like decision tree with Beck Depression Inventory removed from analysis. The model shows the percentage (probability $\times 100$ ) of post-traumatic stress disorder (PTSD), given patient combat experience, REM latency, and paranoid and Wahler Physical Symptom Inventory (WPSI) scores. Red segments show groups of patients with higher probabilities of PTSD than the overall average, whereas green segments show groups of patients with lower probabilities.

Note: Because both patients with and without PTSD are shown, the figure uses the DSM-IV PTSD terms to describe each of the segments.

highlights their importance in the current DSM-5 criteria. This finding highlights the importance of paranoid and hostile behaviour in those with PTSD following a major traumatic experience. ${ }^{6}$ Their presence in these veterans with chronic PTSD, despite their various drug and behavioural treatments, may stem from an inability in civilian life to overcome their combat-ready vigilant mindset, which becomes ingrained in military training. Such cognitive and behavioural abnormalities may contribute to the increased prevalence of dysfunctional domestic and social behaviour in U.S. veterans with PTSD, ${ }^{39}$ and in post-combat British veterans. ${ }^{40}$

In conclusion, the results of this study and previous clinical research support the notion that chronic disturbed sleep physiology, unexplained musculoskeletal pain and fatigue symptoms should be considered to be integral to irremediable post-combat PTSD. Moreover, paranoid and hostile ideation among those with post-combat PTSD, as well as in civilians with $\mathrm{PTSD},{ }^{6}$ may be a cautionary signal to the potential for antisocial behaviour. Further studies are needed to determine whether civilians who are victims of psychologically stressful events (e.g. sexual assault, nonphysically injurious MVA, and industrial and environmental disasters) may demonstrate similar distinguishing features that predispose to PTSD as noted in Fig. 1.

\footnotetext{
Harvey Moldofsky, MD, Dip. Psych., FRCPC, Department of Psychiatry, University of Toronto, Toronto, Ontario, Canada; Lorne Rothman, PhD, SAS (Canada) Institute, Inc, Toronto, Ontario, Canada; Robert Kleinman, MD, Department of Ophthalmology, Stanford University, Palo Alto, California, USA; Shawn G. Rhind, PhD, Individual Behaviour and Performance Section, Toronto Research Centre, Defence Research and Development Canada, Toronto, Ontario, Canada; J. Donald Richardson, MD, FRCPC, Operational Stress Injury Clinic, Parkwood Hospital, London, Ontario, Canada; Department of Psychiatry, Western University, London, Ontario, Canada; Department of Psychiatry \& Behavioral Neuroscience, McMaster University, Hamilton, Ontario, Canada

Correspondence: Harvey Moldofsky, Toronto Psychiatric Research Foundation, 951 Wilson Avenue, Unit 15B, Toronto, Ontario M3K 2A7, Canada. Email: h.moldofsky@utoronto.ca

First received 24 Jun 2015, final revision 16 Oct 2016, accepted 20 oct 2016
}

\section{References}

1 American Psychiatric Association. Diagnostic and Statistical Manual of Mental Disorders (4th edn) (DSM-IV). APA, 1994

2 American Psychiatric Association. Diagnostic and Statistical Manual of Mental Disorders (5th edn) (DSM-5): pp. 271-280. APA, 2013.

3 Palmer IP. War syndromes from 1900 to the present. Symptom patterns and longterm health outcome: pp. 1-27. Report 19990603072 for U.S. Army Medical Research and Materiel Command, Fort Detrick Md., and Royal Defense Medical College, 1999.

4 Moldofsky $\mathrm{H}$. The significance of the sleeping-waking brain for the understanding of widespread musculoskeletal pain and fatigue in fibromyalgia syndrome and allied syndromes. Joint Bone Spine 2008; 75: 397-402.

5 Weathers FW, Keane TM, Davidson JRT. Clinician-administered PTSD scale: a review of the first ten years of research. Depress Anxiety 2001; 13: 132-56.

6 Freeman D, Thompson C, Vorontsova N, Dunn G, Carter L-A, Garety P, et al. Paranoia and post-traumatic stress disorder in the months after a physical assault: a longitudinal study examining shared and differential predictors. Psychol Med 2013; 43: 2673-84.

7 Kamphuis J, Meerlo P, Koolhaas JM, Lancel M. Poor sleep as a potential causal factor in aggression and violence. Sleep Med 2012; 13: 327-34.

8 Freeman D, Stahl D, McManus S, Meltzer H, Brugha T, Wiles $N$, et al. Insomnia, worry, anxiety and depression as predictors of the occurrence and persistence of paranoid thinking. Soc Psychiatry Psychiatr Epidemiol 2012; 47: 1195-203.

9 Derogatis LR, Cleary PA. Confirmation of the dimensional structure of the SCL-90: a study in construct validation. J Clin Psychol 1977; 33: 981-9.

10 Beck AT, Ward CH, Mendelson M, Mock M, Erbaugh J. An inventory for measuring depression. Arch Gen Psychiatry 1961; 4: 561-71.

11 Blanchard EB, Jones-Alexander J, Buckley TC, Forneris CA. Psychometric properties of the PTSD Checklist (PCL). Behav Res Ther 1996; 34: 669-73.

12 Wahler HJ. The physical symptoms inventory: measuring levels of somatic complaining behavior. J Clin Psychol 1968; 24: 207-11.

13 Saskin $\mathrm{P}$, Moldofsky $\mathrm{H}$, Lue FA. Sleep and post-traumatic rheumatic pain modulation disorder (Fibrositis Syndrome). Psychosom Med 1986; 48: 319-23.

14 Moldofsky H, Patcai J. Chronic widespread musculoskeletal pain, fatigue, depression and disordered sleep in chronic post-SARS syndrome; a case-controlled study. BMC Neurol 2011; 37: 11.

15 Hoddes E, Zarcone V, Smythe H, Phillips R, Dement WC. Quantification of sleepiness: a new approach. Psychophysiology 1973; 10: 431-6. 
16 van den Hoed J, Kraemer H, Guilleminault C, Zarcone VP Jr, Miles LE, Dement WC et al. Disorders of excessive daytime somnolence: polygraphic and clinical data for 100 patients. Sleep 1981; 4: 23-37.

17 Rechtshaffen A, Kales A. A Manual of Standardized Terminology, Techniques and Scoring System for Sleep Stages of Human Subjects. UCLA Brain Information Service, 1968.

18 Iber C, Ancoli-Israel S, Chesson A, Quan S, for the American Academy of Sleep Medicine. The AASM Manual for the Scoring of Sleep and Associated Events: Rules, Terminology and Technical Specifications (1st edn). American Academy of Sleep Medicine, 2007.

19 Terzano MG, Parrino L. Clinical applications of cyclical alternating pattern. Physiol Behav 1993; 54: 807-13.

20 Rosa AC, Parrino L, Terzano MG. Automatic detection of cyclic alternating pattern (CAP) sequences in sleep: preliminary results. Clin Neurophysiol 1999; 110: 585-92.

21 Rosa A, Alves GR, Brito M, Lopes MC, Tufik S. Visual and automatic cyclic alternating pattern (CAP) scoring: inter-rater reliability study. Arq Neuropsiquiat 2006; 64: 578-81

22 Rizzi M, Sarzi-Puttini P, Atzeni F, Capsoni F, Andreoli A, Pecis M, et al. Cyclic alternating pattern: a new marker of sleep alteration in patients with fibromyalgia? Rheumatol 2004; 31: 1193-9.

23 Moldofsky $\mathrm{H}$, Harris HW, Archambault WT, Kwong T, Lederman S. Effects of bedtime very low dose cyclobenzaprine on symptoms and sleep physiology in patients with fibromyalgia syndrome: a double-blind randomized placebocontrolled study. J Rheumatol 2011; 38: 2653-63.

24 Bao G, Guilleminault C. Upper Airway Resistance Syndrome-one decade later. Curr Opin Pulm Med 2004; 10: 6

25 Harrar SW, Bathke AC. Nonparametric methods for unbalanced multivariate data and many factor levels. J Multivar Anal 2008; 99: 1635-64.

26 Akritas MJ. The rank transform method in some two-factor designs. J Am Stat Assoc 1990; 85: 409, 73-78.

27 SAS/STAT ${ }^{\circledR}$ 13.1. User’s Guide. SAS Institute Inc., 2013.

28 Hochberg $Y$, Benjamini, Y. More powerful procedures for multiple significance testing. Stat Med 1990; 9: 811-8.

29 Schenker N, Taylor JMG. Partially parametric techniques for multiple imputation. Comput Stat Data Anal 1996; 22: 425-46.
30 Horton NJ, Lipsitz SR. Multiple imputation in practice: comparison of software packages for regression models with missing variables. Am Stat 2001; 55 $244-54$.

31 Argyropoulos SV, Wilson SJ. Sleep disturbances in depression and the effects of antidepressants. Int Rev Psychiatry 2005; 17: 237-45.

32 Kobayashi I, Huntley E, Lavela J, Mellman TA. Subjectively and objectively measured sleep with and without posttraumatic stress disorder and trauma exposure. Sleep 2012; 35: 957-65.

33 Ross RJ, Ball WA, Dinges DF, Kribbs NB, Morrison AR, Silver SM, et al. Rapid eye movement sleep disturbance in posttraumatic stress disorder. Biol Psychiatry 1994; 35: 195-202.

34 Lavie P. Sleep disturbances in the wake of traumatic events. N Engl J Med 2001; 345: $1825-32$

35 Lettieri CJ, Williams SG, Collen JF. OSA syndrome and posttraumatic stress disorder: clinical outcomes and impact of Positive Airway Pressure Therapy. Chest 2016; 149: 483-90.

36 Mellman TA, Nolan B, Hebding J, Kulick-Bell R, Dominguez R. A polysomnographic comparison of veterans with combat-related PTSD, depressed men, and non-ill controls. Sleep 1997; 20: 46-51.

37 Parrino L, Spaggiari M, Boselli M, Giovanni G, Terzano M. Clinical and polysomnographic effects of trazodone $\mathrm{CR}$ in chronic insomnia associated with dysthymia. Psychopharmacology 1994; 116: 389-95.

38 Germain A. Sleep disturbances as the hallmark of PTSD: where are we now? Am J Psychiatry 2013; 170: 372-82.

39 Jakupcak M, Conybeare D, Phelps L, Hunt S, Holmes HA, Felker B, et al. Anger, hostility, and aggression among Iraq and Afghanistan war veterans reporting PTSD and subthreshold PTSD. J Trauma Stress 2007; 20: 945-54.

40 Macmanus D, Dean K, Jones M, Rona RJ, Greenberg N, Hull L, et al. Violent offending by UK military personnel deployed to Iraq and Afghanistan: a data linkage cohort study. Lancet 2013; 381: 907-17. 